\title{
Retenir et reprendre
}

L'écriture dans la pratique de recherche du zoologiste Karl von Frisch

\section{Christoph Hoffmann}

Traducteur : Jean-Louis Lebrave

\section{OpenEdition}

\section{Journals}

Édition électronique

URL : http://journals.openedition.org/genesis/1177

DOI : 10.4000/genesis. 1177

ISSN : 2268-1590

\section{Éditeur :}

Presses universitaires de Paris Sorbonne (PUPS), Société internationale de génétique artistique littéraire et scientifique (SIGALES)

\section{Édition imprimée}

Date de publication : 15 juin 2013

Pagination : 189-205

ISBN : 978-2-84050-893-9

ISSN : $1167-5101$

Référence électronique

Christoph Hoffmann, « Retenir et reprendre », Genesis [En ligne], 36 | 2013, mis en ligne le 06 juillet 2015, consulté le 30 avril 2019. URL : http://journals.openedition.org/genesis/1177 ; DOI : 10.4000/ genesis. 1177 


\title{
Retenir et reprendre L'écriture dans la pratique de recherche du zoologiste Karl von Frisch
}

\author{
Christoph Hoffmann 1
}

\section{Écrire quelque chose}

Si l'on se réfère à la distinction introduite par Roland Barthes, le verbe « écrire » n'est jamais utilisé de manière intransitive dans les sciences « dures ». Les chercheurs n'écrivent pas « absolument », ils écrivent toujours « quelque $\operatorname{chose}^{2} »$. En écrivant et par l'écriture, on développe un argument, on recopie des extraits de la littérature scientifique, on rédige des comptes rendus d'expérience, on fait des calculs, on retient une idée ou un détail surprenant. Toutes ces activités interviennent régulièrement dans la recherche au quotidien, mais elles échappent généralement à l'attention consciente. L'écriture est presque toujours une activité accessoire, étroitement mêlée à d'autres desseins ${ }^{3}$. Si l'on demande à un biologiste ou un physicien ce que leur suggère le verbe écrire, ils parleront très certainement de leurs publications. C'est là, et seulement là, que l'écriture devient pour eux une activité spécifique.

Les réflexions qui suivent portent sur les processus scripturaux qui interviennent pendant la recherche. Ce choix ne signifie évidemment pas que la publication des résultats serait une question dénuée d'intérêt, au contraire. Karin Knorr Cetina et Frederic Holmes ont bien montré la productivité inhérente à l'écriture d'un article 4 . Si cet aspect est mis ici entre parenthèses, c'est dans un but heuristique, pour souligner l'imprévisibilité des processus de recherche. Comme l'écrit Hans-Jörg Rheinberger, «On peut énoncer la formule paradoxale suivante : l'état présent est le résultat de quelque chose qui n'a pas existé sous cette forme, et le passé est la trace de quelque chose qui n'est pas (encore) advenu 5 ». En d'autres termes, la prise en considération de la publication dans l'appréhension du processus de recherche risque de réduire celui-ci à la poursuite d'un but clairement défini à l'avance.
L'objectif de mon article est de montrer comment les processus scripturaux peuvent participer à l'élaboration des objets de recherche. Je m'appuierai sur la pratique de recherche de Karl von Frisch (1886-1982), qui fut longtemps directeur de l'institut de zoologie de l'université de Munich, et plus spécifiquement, sur un épisode de l'été 1944. Contrairement à l'usage actuel, où l'ordinateur est partout, Frisch se sert exclusivement de papier et de crayons. En outre, en tant que zoologiste, il était soumis aux conditions imposées par ses objets de recherche : les observations sur les animaux ne sont pas toujours facilement reproductibles; de plus, elles obligent souvent à travailler sur le terrain. Ce contexte, auquel s'ajoutent des traditions locales, ainsi que des préférences personnelles, marque

1. Je remercie les participants au colloque «Paperworks », qui s'est tenu à Romainmôtier en juin 2012, pour la discussion stimulante d'une première version de ce texte. Un grand merci à Christoph Decker et Jean-Luc Mieusset pour leur soutien et leur aide dans une première traduction de mon texte. Je remercie également la Bayerische Staatsbibliothek de Munich et la famille von Frisch de m'avoir autorisé à publier ces manuscrits.

2. Roland Barthes, «Écrire, verbe intransitif? (1970), dans Le Bruissement de la langue. Essais critiques IV, Paris, Éditions du Seuil, 1984 , p. 21-31, ici p. 28. Mes remarques valent également pour les sciences humaines et sociales, où les chercheurs écrivent sans arrêt mais où nul ne dira spontanément que l'écriture constitue le cœur de son travail.

3. Voir Bruno Latour, « Drawing Things Together », dans Michael Lynch et Steve Woolgar (dir.), Representation in Scientific Practice, Cambridge (Mass.)/London, MIT Press, 1990, p. 19-68, ici p. 21 sq.

4. Voir Karin Knorr Cetina, The Manufacture of Knowledge. An Essay on the Constructivist and Contextual Nature of Science, Oxford/New York, Pergamon Press, 1981, chap. V ; ainsi que Frederic Holmes, dans Scientific Writing and Scientific Discovery, ISIS, ${ }^{\circ} 78,1987$, p. 220-235.

5. Voir Hans-Jörg Rheinberger, Experimentalsysteme und epistemische Dinge. Eine Geschichte der Proteinsynthese im Reagenzglas, Göttingen, Wallstein Verlag, 2001, p. 195. 
fortement la «scène d'écriture ${ }^{6}$ » de Frisch. Il est donc toujours vain d'espérer obtenir des conclusions universellement valables sur l'importance de l'écriture dans le processus de recherche à partir des travaux d'un seul chercheur ou d'un seul groupe de recherche. Cet article vise avant tout à préciser le rôle possible des activités scripturales dans le travail scientifique.

L'histoire des sciences contemporaine connaît deux approches pertinentes pour mon propos. Bruno Latour a souligné l'importance du « paperwork» dans les sciences, et par cette expression il désigne fondamentalement l'accumulation, la mise en relation et la recombinaison de toutes sortes d' « inscriptions 7 ». Ursula Klein, quant à elle, introduit la notion de «paper tool», qui renvoie à un système de notations (dans son cas, il s'agit de formules chimiques) dont l'utilisation permet aux phénomènes de prendre forme et d'être analysés ${ }^{8}$. Les approches de Latour et de Klein se rejoignent en ce qu'elles mettent l'accent sur ce qu'on fait « avec du papier » et « sur le papier ». Mes propres réflexions s'en inspirent et visent à renforcer l'instrumentalité propre à ces activités scripturales en introduisant la notion de procédure d'écriture. Celle-ci désigne une suite plus ou moins stabilisée d'étapes dans laquelle des entités (mots, chiffres, phénomènes abstraits ou questions et problèmes ouverts) sont traitées ${ }^{9}$. D'une manière générale, on peut distinguer deux types de procédures d'écriture : celles où les entités sont ordonnées dans des configurations spatiales; la liste en fournit un exemple simple ${ }^{10}$. Et celles où le traitement des entités est intégré dans des séquences temporelles; un exemple simple en est le recopiage. Ces procédures peuvent en outre être héritées d'une tradition culturelle, comme par exemple l'annotation dans les marges. Mais elles peuvent aussi relever des pratiques individuelles.

Les documents du fonds d'archive de Frisch permettent d'analyser aussi bien son travail « sur le papier » que son travail « avec du papier ». Particulièrement intéressants sont ses journaux d'observations et les regroupements annuels d'expériences confectionnés à partir de ces journaux. Ces matériaux semblent presque complets pour la période allant de 1944 au début des années soixante. Le présent article portera essentiellement sur la relation systématique entre ces journaux et ces regroupements annuels. C'est pourquoi je n'aborderai pas le détail des séquences temporelles et des arrangements spatiaux sur les différents feuillets, mais m'attacherai plutôt à dégager des caractéristiques générales. Pour ce faire, j'utiliserai un troisième corpus qui, sous le titre, peut-être ajouté après coup, de «thèmes », rassemble des questions de recherche. Ce travail suggère que chez Frisch, l'écriture fonctionne comme une procédure récursive (dans le temps). Écrire présuppose toujours que le scripteur reviendra ultérieurement sur le déjà écrit.

\section{Consigner des observations}

Comme la plupart des zoologistes, Karl von Frisch avait un « animal préféré ». Dans son cas, c'était l'abeille, dont il a étudié les capacités sensorielles du début de sa carrière, avant la Première Guerre mondiale, jusqu'à ce qu'il se retire dans les années soixante. Il s'est intéressé à la perception des couleurs et des odeurs chez les abeilles, à leur orientation dans l'espace et à leur système de communication. Ce sont plus particulièrement les travaux sur le thème de la communication par la danse chez les abeilles qui ont été discutés bien au-delà du cercle des spécialistes. Aujourd'hui encore, ils sont déterminants pour l'état actuel de la recherche ${ }^{11}$. Frisch a reçu le prix Nobel pour l'ensemble de son œuvre en 1973 en même temps que Konrad Lorenz et Niko Tinbergen.

6. Voir Rüdiger Campe, «Die Schreibszene. Schreiben », dans Paradoxien, Dissonanzen, Zusammenbrüche. Situationen offener Epistemologie, Hans Ulrich Gumbrecht et K. Ludwig Pfeiffer (dir.), Frankfurt/Main, Suhrkamp, 1991, p. 759-772.

7. Voir Bruno Latour, « Drawing things together », art. cit., p. 52-60.

8. Voir Ursula Klein, « Paper Tools in Experimental Cultures - The Case of Berzelian Formulas », Studies in History and Philosophy of Science, $\mathrm{n}^{\circ} 32,2001$, p. 265-312.

9. Voir Christoph Hoffmann, « Schreiben als Verfahren der Forschung », dans Michael Gamper (dir.), Experiment und Literatur: Themen, Methoden, Theorien, Göttingen, Wallstein Verlag, 2010, p. 181-207.

10. Voir Jack Goody, The Domestication of the Savage Mind, Cambridge/ New York, Cambridge University Press, 1977, ici p. 74-111.

11. Voir Tania Munz, «The Bee Battles: Karl von Frisch, Adrian Wenner, and the Honey Bee Dance Language Controversy », Journal of the History of Biology, $\mathrm{n}^{\circ} 38,2005$, p. 535-570 ; ainsi que The Dancing Bees. Karl von Frisch, the Honeybee Dance Language, and the Sciences of Communication, Chicago (parution automne 2013). 
Dans ce qui suit, je vais me concentrer sur les notes prises dans une phase centrale des recherches sur le « langage de la danse chez les abeilles ». Frisch avait publié un premier récapitulatif de ses travaux au début des années vingt ${ }^{12}$, puis il s'était consacré à d'autres domaines de recherche. Le travail sur les abeilles reprit de l'importance pendant la Seconde Guerre mondiale en raison des efforts entrepris pour améliorer l'approvisionnement en produits agricoles. C'est sur cette question que portent les recherches consignées dans les premières pages du journal d'observations «Brunnwinkl 1944 III'13 ». D'après la désignation qui figure sur la page de couverture, il s'agit du troisième des cinq journaux rédigés cette année-là ; il contient les observations faites à partir d'août 1944 par Frisch dans sa résidence d'été de Brunnwinkl au bord du lac Wolfgangsee (Autriche). À cette époque, Frisch a étudié la possibilité de dresser les abeilles à polliniser certaines plantes en ajoutant des parfums à leur alimentation. Une réserve de nourriture était placée à côté de la ruche, quelques-unes des abeilles qui approchaient la nourriture étaient marquées (les autres par contre étaient tuées), puis on observait le comportement des abeilles marquées dans la ruche ; un peu après, on notait le nombre d'abeilles qui se présentaient à un poste d'observation situé lui aussi près de la ruche, où on avait placé la même nourriture. Ces travaux prirent une tournure imprévue dont l'élément déclencheur fut une variante dans la construction de l'expérience. Au lieu de placer comme précédemment la source de nourriture à dix mètres de la ruche, on la mit plus loin, à cent quarante mètres. On s'attendait à ce que les abeilles prévenues dans la ruche commencent par chercher la nourriture près de la ruche, puis la recherchent de plus en plus loin. On avait mis en place deux postes d'observation pour contrôler l'expérience, l'un près de la ruche, l'autre près de la source de nourriture (plus éloignée de la ruche que dans l'expérience précédente).

Le résultat fut spectaculaire. Frisch raconte dans son autobiographie : « La coupelle placée près de la ruche ne les intéressait guère, mais la coupelle éloignée fut bientôt entourée d'un essaim d'abeilles. Y avait-il donc dans leur "langue" un mot pour signifier l'éloignement14 ? ${ }^{14} \mathrm{Au}$ terme d'une série de découvertes déclenchées par ce résultat, il est apparu qu'en effectuant des danses dans la ruche, les abeilles peuvent communiquer non seulement la distance, mais aussi la direction dans laquelle se trouve une source de nourriture ${ }^{15}$. L'observation initiale a été datée par Frisch du 12 août 1944. Examinons les notes correspondantes dans son journal d'observations. D'abord, il apparaît que les chiffres ne sont pas aussi univoques qu'on pourrait le penser à la lecture de l'autobiographie : au poste d'observation A, près de la ruche, on compte vingt-neuf approches; on en compte trente-huit au poste d'observation B, près de la source de nourriture (voir fig. 1, à droite $)^{16}$. Cependant, le plus intéressant pour mon propos est que ces deux pages ont retenu les événements survenus tout autour de la ruche.

D'abord, que veut dire exactement « retenir » dans ce contexte ? Pour décrire les effets produits par le cahier de laboratoire, Odile Welfelé recourt à la formule « organiser le désordre ${ }^{17} »$. Au cours du processus par lequel elles sont notées, un grand nombre d'impressions et d'actions sont placées dans un ordre schématique. De même, Hans-Jörg Rheinberger parle d' « effets de condensation »: «Au sens le plus trivial, cela signifie que l'arrangement spatial et temporel de l'expérience, avec ses données disséminées dans les quatre dimensions, est essentiellement mis à plat et copié sur une surface à deux dimensions ${ }^{18}$. » Dans le cas qui nous occupe, voici ce qui est retenu des événements survenus autour de la ruche (fig. 1) : sur la page de gauche, la date du jour d'observation, les conditions météorologiques, la localisation de la source de nourriture, sa nature et sa quantité, la durée de la prise de nourriture, le numéro des abeilles présentes à la source de nourriture, le nombre

12. Karl von Frisch, "Über die "Sprache" der Bienen. Eine tierpsychologische Untersuchung », Zoologische Jahrbücher: Abteilung für allgemeine Zoologie und Physiologie der Tiere, $\mathrm{n}^{\circ}$ 40, 1923, p. 1-186. 13. Fonds Karl von Frisch, Ana 540, A III, 1944 III, Bayerische Staatsbibliothek München, collection de manuscrits.

14. Karl von Frisch, Mémoires d' un biologiste. Le professeur des abeilles (1973), trad. Michel Martin et Jean-Paul Guiot, Paris, Belin, 1987 (3e éd.), p. 175 .

15. Voir Karl von Frisch, Tanzsprache und Orientierung der Bienen, Berlin/Heidelberg/New York, Springer Verlag, 1965.

16. Voir fonds Karl von Frisch, Ana 540, A III, 1944 III, f $\mathrm{f}^{\circ} 12 \mathrm{v}^{\circ}-13 \mathrm{r}^{\circ}$.

17. Odile Welfelé, « Organiser le désordre : usages du cahier de laboratoire en physique contemporaine », Alliage, n 37/38, 1998-1999, p. 25-41, p. 32.

18. Hans-Jörg Rheinberger, « Scripts and Scribbles », Modern Language Notes, $\mathrm{n}^{\circ} 118,2003$, p. 622-636, ici p. 625. 


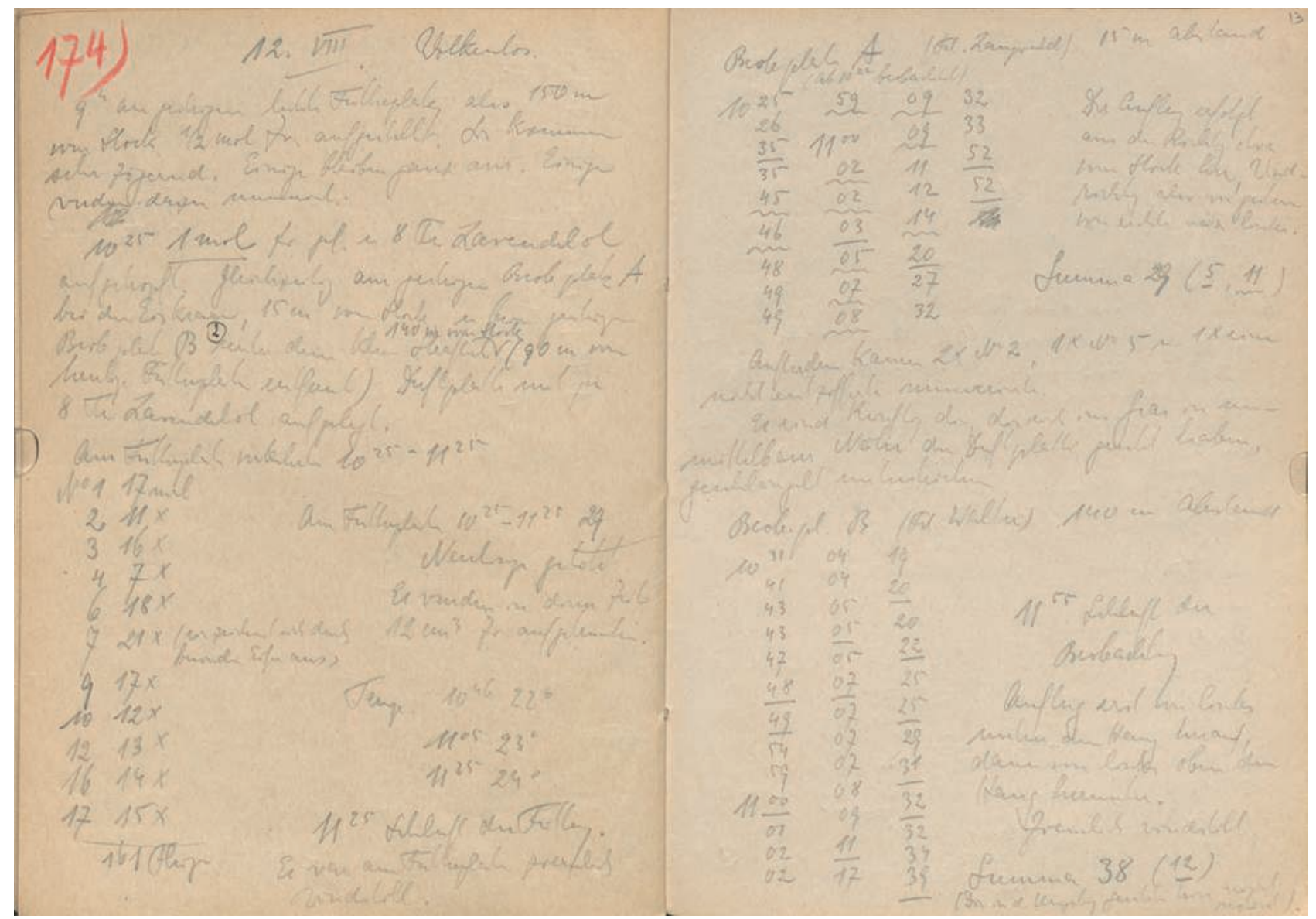

Fig. 1 : Journal d'observations de Karl von Frisch, notes prises le 12 août 1944. À gauche dans la partie supérieure, en rouge, le numéro de l'expérience (174) ajouté postérieurement ; à droite le résultat du décompte dans deux postes d'observation différents (Bayerische Staatsbibliothek, collection de manuscrits, Ana 540, A.III, 1944 III. Tous droits réservés @ Bayerische Staatsbibliothek, München, \& famille von Frisch)

d'abeilles «nouvelles » qui y sont tuées, la température et la direction du vent à l'endroit où se trouve la réserve de nourriture ; sur la page de droite, pour chacun des deux postes d'observation, le nom des observatrices, les heures d'arrivée des abeilles, leur nombre total, la direction principale de l'approche ainsi que les données concernant le vent. Par contre, on ne trouve aucune mention des autres événements survenus à proximité de ces différents points ; surtout, il n'y a rien sur la logique de la démarche : il n'est par exemple pas non plus mentionné pourquoi la source de nourriture a été éloignée de la ruche.

Retenir va donc de pair avec des omissions considérables. Il faut dans ce cas en chercher les raisons dans les intérêts du chercheur : Frisch décide de ce qui est important, de ce qui est sans intérêt, de ce qui, étant évident, n’a pas besoin d'être noté. Lorsqu'on lit le journal d'observations pour reconstruire le processus de recherche, on se trouve face à une partie assez fragmentaire des événements qui se sont déroulés autour de la ruche. Mais cela vaut également pour Frisch lui-même. Pour lui aussi, ne subsiste du comportement des abeilles que ce qui a trouvé place dans les cahiers. Il apparaît donc que tout ce qui est noté par écrit - et plus encore tout ce qui n'est pas noté - conditionne l'avenir. Les notes recueillies dans le journal d'observations «préforment » ce qu'il sera possible de tirer des événements du 12 août 1944. Si l'on examine à nouveau les éléments qui ont été consignés, on voit que malgré leur caractère factuel (il s'agit fondamentalement de chiffres et de données météorologiques), ce sont avant tout des potentialités. Toutes ces données pourront ultérieurement devenir 
importantes pour la compréhension des événements qui se sont déroulés autour de la ruche. Mais à ce stade de la notation, il n'est pas encore possible de dire si elles ont vraiment une signification. Dans cette perspective, retenir des observations signifie, en fin de compte, configurer à l'avance les possibilités de connaissances futures.

\section{Élaborer des résultats}

Le journal d'observations de Frisch constitue un chaînon dans une séquence d'enregistrements. Il doit d'ailleurs être lui-même la réélaboration d'autres enregistrements puisque les données ont été recueillies simultanément dans deux postes d'observation différents. Et l'étape suivante a laissé elle aussi des traces dans le journal. Reprenons les pages du journal datées du 12 août 1944 : dans le coin en haut à gauche se trouve le nombre 174 , écrit dans la même écriture rouge que l'indication « V 137 - $179 »$ sur la couverture du journal ${ }^{19}$. Ces chiffres correspondent à des «numéros d'expériences » attribués rétrospectivement par Frisch, comme le montre le fait qu'ils sont parfois écrits un peu en surcharge sur les inscriptions du journal. Notons que les journaux ne comportent pas d'indications sur la logique qui a présidé aux recherches. Il n'est pas noté si les observations consignées quotidiennement ont un lien entre elles, et si oui, quel est ce lien ; fait encore plus remarquable, on ne trouve nulle part d'indication sur l'objet de la recherche. Si nous n'avions que ces cahiers, personne - à l'exception peut-être de Frisch lui-même - ne pourrait reconstituer entièrement l'intention qui a présidé à la mise en œuvre des processus que nous y découvrons. Cette situation ne change que si l'on suit le chemin tracé par les numéros rouges des expériences.

Le fonds d'archives de Frisch contient un dossier dont la couverture porte, écrite avec le même crayon rouge que nous avons déjà rencontré, l'inscription « registre des numéros d'expérience », ainsi que la même inscription, mais en petits caractères et au crayon noir, accompagnée de l'ajout «1946 - 1950 » (en fait, les matériaux vont de 1944 à 1964) ${ }^{20}$. Le dossier contient environ cinquante feuilles volantes de dimensions proches du format A4 qui sont toutes rédigées de manière identique. En haut, l'indication de la date constitue le titre ; elle est complétée à partir de 1946 par l'indication des «numéros de registre »; en dessous, on trouve une liste en forme de tableau. À gauche il y a quelques mots, le plus souvent soulignés, à droite des suites de chiffres. En tête de la liasse se trouve le feuillet pour l'année 1944, où l'on trouve l'expérience 174 dès la première série de numéros ; elle porte le titre « Expériences d'alimentation à différentes distances et directions » (voir fig. 2)21. Rien de très spectaculaire en apparence. Pourtant, il s'est produit quelque chose d'une importance considérable. Liées les unes aux autres par le numéro d'expérience, les observations consignées quotidiennement dans le journal d'observations sont regroupées, mises en relation, et surtout rapportées à un thème particulier, ce qui crée une articulation en une série d'expériences spécifiques. La question soulevée par la lecture du journal trouve ainsi sa réponse : les observations du 12 août 1944 font partie des expériences dans lesquelles on faisait varier la distance et/ou la direction de la source de nourriture.

Avec la mise en place d'un registre des numéros d'expérience, les observations du journal d'observations, dont le rapport qu'elles entretenaient entre elles et la finalité n'étaient pas explicitées, prennent place dans une première structure de signification : certaines des observations quotidiennes se révèlent être des expériences sur le changement de distance et/ou de direction, celles qui figurent dans le paragraphe suivant du registre des numéros sont des expériences dans lesquelles la glande à parfum a été obturée, celles du paragraphe suivant portent sur la « forme de la danse », etc. Il n'est pas possible de déterminer directement quand cette classification a été réalisée. Mais si l'on se souvient que la numérotation des expériences a été ajoutée a posteriori dans le journal, l'idée s'impose que le but et le contexte du travail quotidien n'ont été fixés que d'une manière récursive, dans et par la confection du registre. Cela ne veut pas dire que pendant tout l'été 1944, Frisch a travaillé à l'aveuglette et sans but précis. Il faut

19. Voir Fonds Karl von Frisch, Ana 540, A III, 1944 III, couverture. 20. Fonds Karl von Frisch, Ana 540, A IV, 2, couverture, Bayerische Staatsbibliothek München, collection de manuscrits.

21. Ibid., folios non numérotés, [Versuchsnummernprotokoll für das Jahr 1944, première feuille]. 


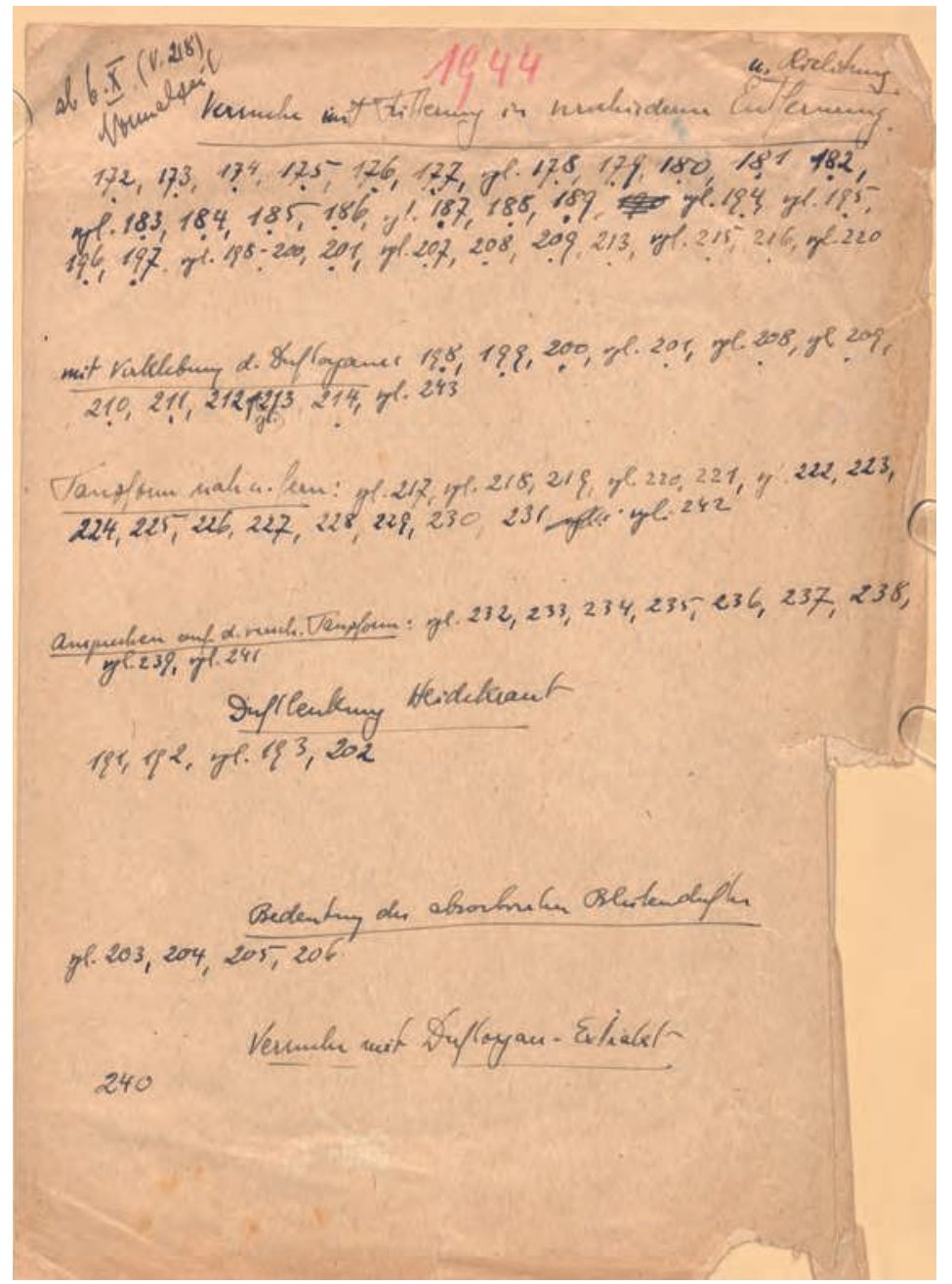

Fig. 2 : «Registre des numéros d'expérience » pour l'année 1944. En dessous du titre « Expériences d'alimentation à différentes distances et directions », dans le premier bloc de chiffres, en troisième position, le numéro de l'expérience 174

(Bayerische Staatsbibliothek, collection de manuscrits, Ana 540, A.IV, 2.

Tous droits réservés (C) Bayerische Staatsbibliothek, München, \& famille von Frisch)

bien plutôt considérer qu'en créant un registre des expériences, il transforme une démarche implicite, et peutêtre aussi intuitive, en un système expérimental formulé explicitement. En outre, ce registre marque aussi une réorientation durable dans la manière d'envisager les phénomènes observés.

C'est ce que suggèrent quelques feuilles volantes placées à la fin du cinquième et dernier journal d'observations de l'année 1944. Frisch y revient sur une série d'observations qu'il a faites à partir d'août 1944. Se servant des notes prises dans le journal, il reprend la position des sources de nourriture et des deux points d'observation sous forme d'une esquisse à deux dimensions, il y ajoute ensuite les distances entre chaque point et le nombre d'abeilles comptées aux postes d'observation. Le feuillet du 12 août comporte un petit commentaire écrit à l'encre dans la marge inférieure (voir fig. 3) : «Bien que le vent pousse l'odeur de la source de nourriture proche vers la ruche, il vient bien moins d'abeilles que dans les deux premières expériences. L'huile ess.[entielle] seule ne peut donc être la cause du nombre important de visites à la source de nourriture proche ${ }^{22}$. » Certaines inconséquences dans ces

22. Fonds Karl von Frisch, Ana 540, A III, 1944 V, feuilles intercalées, $\mathrm{f}^{\circ} 3 \mathrm{r}^{\circ}$, Bayerische Staatsbibliothek München, collection de manuscrits. 


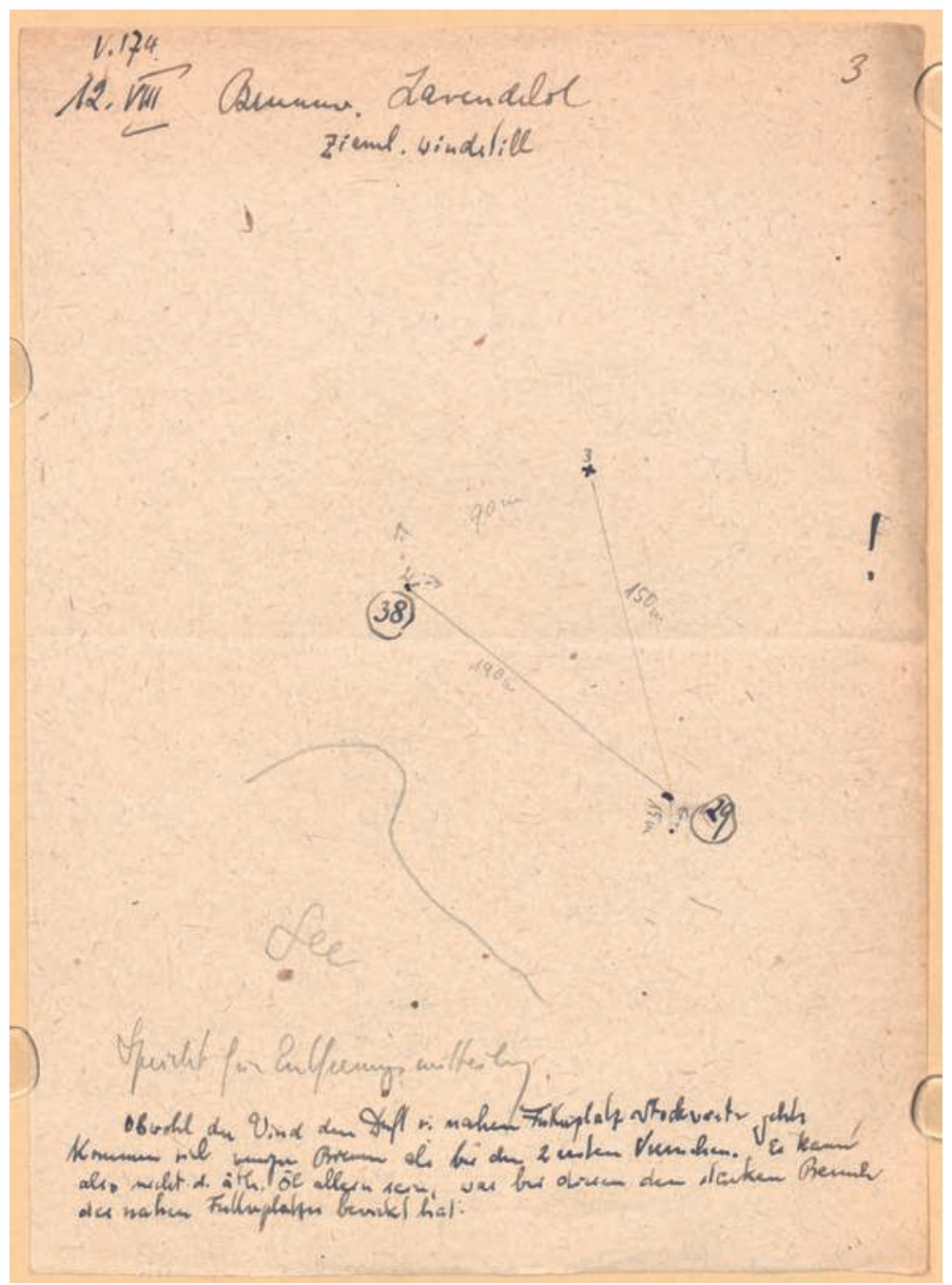

Fig. 3 : Esquisse sur feuille volante se rapportant à l'expérience $n^{\circ} 174$, datant probablement de la fin septembre ou du début octobre 1944

(Bayerische Staatsbibliothek, collection de manuscrits, Ana 540, A.III, 1944 V.

Tous droits réservés @ Bayerische Staatsbibliothek, München, \& famille von Frisch)

notations ${ }^{23}$ suggèrent que la pensée est encore en pleine élaboration au moment où elle est mise sur le papier. De toute évidence, l'appréhension des phénomènes observés connaît une réorientation. L' « huile ess.[entielle] seule », ou plus exactement son « odeur » ne peut pas, dit Frisch, avoir provoqué la recherche d'une source de nourriture par les abeilles. La note écrite au crayon papier au-dessus de ce commentaire renforce encore cette considération : «Suggère une communication des distances 24 . »

Le fac-similé numérique ne permet pas de savoir dans quel ordre ces deux notes ont été écrites. Mais il y a un autre indice chronologique tout aussi intéressant. Dans le coin supérieur gauche de la feuille, un simple «V. 174 » est écrit au-dessus de la date du «12. VIII », elle-même suivie d'une mention servant de titre « Brunnw.[inkl] huile de lavande pratiquement sans vent » (fig. 3). L'esquisse et

23. Ainsi, Frisch écrit « source de nourriture » (Futterplatz) au lieu de «poste d'observation »(Beobachtungsplatz). De même, le texte se contredit d'une phrase à l'autre : il dit d'abord que moins d'abeilles viennent à la source de nourriture proche (poste d'observation), puis il souligne le grand nombre de visites à cette même source de nourriture proche.

24. Fonds Karl von Frisch, Ana 540, A III, 1944 V (Anm. 22), feuilles intercalées, $\mathrm{f}^{\circ} 3 \mathrm{r}^{\circ}$. 
les commentaires qui l'accompagnent sont donc postérieurs à la numérotation des expériences. En outre, les feuillets ont été placés dans le dernier journal de l'année 1944. Ils n'ont donc probablement pas été rédigés avant la fin de la période de recherche, soit octobre 1944. Cela confirme l'idée que la structure de significations à laquelle Frisch intègre son travail est postérieure aux notes faites sur le terrain - elle court en quelque sorte derrière elles. L'idée que les abeilles ont un « mot pour la distance », comme l'écrit Frisch dans son autobiographie, n'aurait donc acquis son importance que d'une manière progressive, à travers une série de notations successives.

Pour apprécier ce processus, on peut d'abord rappeler l'affirmation de Latour selon laquelle le «paperwork» peut produire des phénomènes totalement nouveaux par une recombinaison et une synthèse des notes 25 . Dans les grandes lignes, cela vaut certainement pour la pratique de recherche de Frisch. Toutefois, il me semble que l'élément déterminant n'est pas tant le fait que quelque chose de nouveau prenne forme au cours du travail « avec des papiers », que la relation que cet effet entretient avec les notes. Il est évident que tout ce qui se passe est causé d'abord par le déplacement de la source de nourriture, quelle qu'en soit la raison. Mais la manière dont Frisch a organisé son «paperwork » confère à l'activité de recherche un effet d'après-coup. On peut aller jusqu'à dire qu'elle anticipe la venue de cette intelligence d'après-coup dès le moment de la recherche. La succession du journal de travail, de la numérotation des expériences et du registre des expériences constitue ainsi la condition de possibilité d'un style de recherche dans lequel les observations effectuées un jour donné suscitent les investigations du jour suivant, tandis que la structure de signification proprement dite n'apparaît que dans l'élaboration ultérieure des données stockées dans le journal.

Ce processus n'est pas linéaire : le feuillet qu'on vient de commenter (fig. 3) ne témoigne pas vraiment d'une compréhension plus profonde des observations consignées dans le journal d'observations. Il est plutôt le témoin d'un moment de déstabilisation, au sein duquel, d'une manière plutôt hésitante et quelque peu chaotique, se fait jour un changement fondamental dans la compréhension des événements. Contrairement à ce que Frisch supposait dès les années vingt ${ }^{26}$, l'odeur des fleurs paraissait ne pas jouer le premier rôle dans la transmission des informations à l'intérieur de la ruche. De même, le registre des numéros d'expérience ne fournit pas une théorie complètement élaborée des phénomènes étudiés, mais il dresse la liste des catégories sous lesquelles les travaux de l'année 1944 seront identifiés désormais comme des expériences. Chacun des mots-clés placés devant les blocs de nombres représente lui aussi des potentialités. La question reste ouverte de savoir si le regroupement des expériences sous les motsclés « distance » et « direction », « obturation de la glande à parfum », «forme de la danse pour le proche et pour le lointain » ou, plus bas, "réponse à la forme de danse ronde » (fig. 2) acquiert un sens stable 27.

\section{Trouver des questions}

Le fonds d'archives de Frisch est particulièrement riche. En plus des documents déjà mentionnés, il contient de très nombreuses correspondances, des manuscrits de conférence, quelques photos et une série de plus de trente recueils d'extraits. Ces derniers sont regroupés par thème, ainsi « sens de la lumière et des couleurs », «biologie » ou « abeilles », et leur consultation est facilitée par un index. Ces extraits ont une caractéristique remarquable : qu'il ait seulement noté un détail ou recopié un extrait de grandes dimensions, Frisch ne fait généralement ni commentaire (qu'il soit positif ou négatif) ni allusion à un rapport avec ses recherches en cours. Bien sûr, les domaines d'activité principaux de Frisch se reflètent dans le classement thématique. Cependant, les extraits ne fournissent aucun indice direct quant à l'existence d'un « filtre de lecture » supplémentaire, plus fin, qui présiderait aux choix bibliographiques. Comme pour les notes recueillies dans le journal d'observations, rien n'indique quels intérêts particuliers ont présidé au choix des extraits, ni même si un tel intérêt associé à un objet de recherche concret existe.

25. Voir Latour, « Drawing Things Together », art. cit., p. 60.

26. Voir von Frisch, « Über die "Sprache” der Bienen», art. cit., p. 174. À cette époque, Frisch suppose par ailleurs que les abeilles ne portent pas seulement l'odeur des fleurs dans la ruche mais marquent aussi la source de nourriture avec leur propre organe odorant.

27. Fonds Karl von Frisch, Ana 540, A IV, 2, folios non numérotés [Versuchsnummernprotokoll für das Jahr 1944, première feuille]. 
Globalement, il semble que Frisch ait soigneusement veillé à préserver la séparation des différents aspects de son travail telle qu'elle se manifeste dans les différentes séries d'enregistrements (journaux de travail, registre des numéros d'expérience, recueil d'extraits). Un élément appelé «Thèmes » dans le catalogue du fonds le suggère. Le titre est particulièrement intéressant si l'on se souvient que dans tous les documents directement liés à sa pratique de la recherche, Frisch ne parle jamais de ses idées ou des intentions qui le guident. Or, ce titre correspond, non pas à un de ces cahiers d'écolier si souvent utilisés par Frisch, mais à un gros classeur contenant une quantité impressionnante de petits morceaux de papier couverts d'écriture. Le plus souvent, ce sont des fragments soigneusement découpés dans des feuilles de papier à lettres, des manuscrits, des pages de cahier, mais c'est aussi le verso de vieilles cartes de visite, ou parfois une page de correspondance. Voici quatre exemples tirés de cette masse de plus de deux cents billets : «Tr.[avail] d'admission au diplôme ou de doctorat. L'organe vocal des abeilles d'eau », «Est-ce que les poissons marins perçoivent le goût salé ? (dressage ?) », « Dans la volière du nouvel institut : est-ce que des charges apparaissent sur le plumage des oiseaux ? év.[entuellement] pendant le vol ? chez des oiseaux apprivoisés, par ex. des perruches », «Sujet pour un tr. [avail] d'admission au diplôme : Marquer les abeilles en extérieur et voir si ce sont toujours les mêmes abeilles qui vont sur la même fleur, de telle sorte que, comme le facteur relevant le courrier, chaque abeille aurait son propre itinéraire, qui pourrait ne pas être très long. Essayer de déterminer totalement cet itinéraire 28 ».

En définitive, c'est donc ici, dans cette pile de petits papiers, qu'apparaît ce qu'on cherche vainement partout ailleurs : des plans, des intentions, des hypothèses griffonnées en quelques mots. Mais ce qui est caractéristique, c'est qu'il s'agit d'une réserve de thèmes non ordonnés. En lieu et place d'une cohérence, on serait tenté de dire, d'une épistémè, on rencontre un capharnaüm de questions. Bien sûr, cela correspond sur le plan institutionnel aux besoins d'un professeur d'université qui doit distribuer des sujets de thèse. Mais par-delà cette donnée factuelle, ce réservoir thématique reproduit une caractéristique de tous les recueils de notes de Frisch. Beaucoup de ces billets renvoient à des références bibliographiques ou à des extraits de publications, d'autres paraissent être des notes à usage personnel. Au fond, on pourrait parler de trouvailles et d'idées qui viennent à Frisch en passant et qui dépendent du contexte : à l'occasion d'une lecture, dans la vie quotidienne (les abeilles et le facteur), ou au vu des ressources disponibles (les volières du nouvel institut). L'enjeu de cette collection serait le fait lui-même de collectionner, bien plus que les thèmes recueillis - à propos desquels on est presque tenté de parler d'épluchures... En d'autres termes, même la « découverte de thèmes » prend place chez Frisch au sein d'une série de notes qui certes renvoie partiellement aux recueils d'extraits, mais dont l'ensemble ne fait pas apparaître une organisation cohérente au niveau du contenu. Les « thèmes » ne sont pas des aspects d'un programme de recherche d'ensemble - du moins, ce n'est pas ainsi qu'ils ont été organisés par Frisch. Quant à savoir si toutes les questions soulevées ont été abordées, le problème reste ouvert. L'économie générale d'une telle réserve suggère plutôt qu'il s'agit d'un résidu de questions qui n'ont pas été traitées. Les notices portant des sujets effectivement exploités ont probablement été retirées.

\section{Une écriture tournée vers l'avant}

Tout bien considéré, on trouve simultanément chez Frisch une importante programmation de l'usage du crayon et du papier et une sous-détermination remarquable du contenu concret des annotations. L'organisation de « l'activité d'écriture » laisse largement ouverte la finalité des annotations. On peut dire aussi que par elle, la structure de signification ne se met en place que d'une manière progressive, dans et par la succession des annotations. À l'instant où la note est rédigée, aucun choix n'a encore été fait sur le moment où les thèmes notés seront étudiés ou sur la personne qui mènera l'étude. Les extraits tirés de la littérature ne paraissent pas fonctionner comme des références ou des suggestions pour la recherche en cours. C'est seulement lorsque l'exploitation des journaux de travail est rendue possible par les registres des numéros d'expériences que se forme un système de catégories qui

28. Fonds Karl von Frisch, Ana 540, A VII, 37, folios non numérotés, Bayerische Staatsbibliothek München, collection de manuscrits. 
permet d'appréhender les observations consignées comme des « observations de quelque chose ». Dans un de ses Cahiers, Paul Valéry a noté la pensée suivante : «Je n'arrive pas à ce que j'écris, mais j'écris ce qui conduit où ?29 » Cette phrase énonce en quelque sorte la règle utilisée par Frisch. Chez Frisch, contrairement aux apparences et bien que ce soit en même temps aussi le cas, « écrire quelque chose » (Roland Barthes) ne signifie pas simplement mettre une chose sur le papier. C'est d'abord écrire vers l'avant, vers l'avenir, et en présupposant qu'il y aura d'autres phases de travail. À la différence de ce qu'écrit Valéry, il semble toutefois que chez Frisch, ce ne soit pas tant l'écriture que la reprise du déjà écrit qui ouvre la voie vers l'avenir.

Anouk Barberousse a caractérisé les griffonnages que les chercheurs produisent si souvent dans leur travail comme des « raisonnements en acte 30 ». Dans le fonds d'archives de Frisch, ces griffonnages sont presque totalement absents. La série de feuilles volantes évoquée plus haut, dans laquelle il reprend à l'automne 1944 les observations qu'il a faites à partir du mois d'août de la même année (fig. 3), constitue plutôt une exception. Surtout, elles sont elles aussi prises dans un mouvement récursif. Ce qui est noté, ce n'est pas ce qui se passe au moment présent mais ce qui s'est déroulé plusieurs semaines auparavant. Pour décrire la pratique de recherche de Frisch, on pourrait donc dire qu'il raisonne en assemblant et en reprenant de l'écrit. «Où cela conduit », pour reprendre Valéry, cela reste réellement ouvert, mais est en même temps contraint par sa place dans l'organisation des enregistrements et leur enchaînement. Dans ce sens spécifique, écrire est ici un instrument de recherche, c'està-dire une procédure qui consiste fondamentalement à reporter de manière planifiée des significations et des intentions. Cette procédure n'a pas de nom particulier, elle ne fait pas partie des procédures collectives comme les listes, les diagrammes, les annotations ou les synopsis. Elle est sans doute difficilement dissociable de la personne de Frisch, et il n'est même pas certain qu'il l'ait perçue explicitement comme procédure. Cependant, on peut l'appréhender analytiquement comme une régularité qui produit des effets spécifiques - elle comporte donc les traits distinctifs qui caractérisent toute procédure. En sciences (mais pas seulement en sciences), l'écriture possède une réalité propre. Elle ne se résout pas dans l'activité de pensée ; on ne peut non plus la réduire à sa fonction de stockage. C'est cette réalité qu'il convient d'étudier - on peut, pour y parvenir, utiliser la notion de procédure comme une sonde lancée dans les fonds manuscrits.

Traduit de l'allemand par Jean-Louis Lebrave

29. Paul Valéry, Cahiers, édition établie, présentée et annotée par Judith Robinson, Paris, Gallimard, 1973, vol. I, p. 7.

30. Anouk Barberousse, «Dessiner, calculer, transmettre : écriture et création scientifique chez Pierre-Gilles de Gennes », Genesis, n² 20, «Écriture scientifique », 2003, p. 145-162, ici p. 155. 
Christoph Hoffmann est professeur d'épistémologie (Wissenschaftsforschung) à l'Université de Lucerne. Parmi les thématiques de ses recherches, on retiendra le rôle tenu par l'écrire dans les sciences ainsi que l'expérimentation animale au xxe siècle. Publications concernant ces contextes de recherche : Christoph Hoffmann (dir.), Daten sichern. Schreiben und Zeichnen als Verfahren der Aufzeichnung, Berlin, Diaphanes, 2008 ; « Processes on Paper: Writing Procedures as non-material research devices », Science in Context, vol. 26, 2013, no 2 ; «The ruin of a book: Jean André de Luc's "Recherches sur les modifications de l'atmosphère" (1772) », Modern Language Notes, vol. 118, 2003, p. 586-602.

christoph.hoffmann@unilu.ch

Résumés

\section{Retenir et reprendre. L'écriture dans la pratique de recherche du zoologiste Karl von Frisch}

Écrire est l'une des activités les plus ordinaires des savants. Je me propose de considérer le rôle que peuvent jouer les notes dans le processus de recherche en étudiant les travaux du zoologiste autrichien Karl von Frisch (1886-1982), dont la définition de la « danse des abeilles » n'a en rien perdu de son actualité. Ce sont les données obtenues en été 1944 qui ont amené von Frisch à modifier sensiblement sa conception du langage des abeilles. Cette rupture conceptionnelle ne s'est produite, cependant, que dans (et par) la transformation de notes copiées et réécrites à partir de notes prises lors de l'observation des abeilles. Von Frisch semble donc s'être servi de l'écriture pour déterminer après coup la signification concrète demeurée indéterminée dans des notes produites antérieurement. Cet exemple illustre d'une façon plus générale que l'écrit (du relevé d'observations à l'ébauche ultime d'une publication) constitue l'un des instruments associés à la genèse de la connaissance scientifique.

W riting is one of the most ordinary activities of scientists. I will evaluate the role that notes play in the research process by studying the works of the Austrian zoologist Karl von Frisch (1886-1982), whose definition of "bees' dance" is as topical as ever. It is this data obtained during the summer of 1944 that led von Frisch to considerably modify his ideas about bees' language. However, this conceptual breakthrough was made possible only by the transformation of notes copied and rewritten from notes taken down during the observation of bees. It thus seems that Von Frisch used writing to determine after the fact the concrete significance that had remained indeterminate in the previous notes he had taken. This example illustrates in a more general way that writing (from observation notes to the final draft for publication) constitute one of the instruments associated with the genesis of scientific knowledge.

Schreiben gehört auch in den Naturwissenschaften zu den alltäglichsten Beschäftigungen. In diesem Aufsatz möchte ich überlegen, welche Rolle schriftliche Aufzeichnungen im Forschungsprozess spielen können. Ich beziehe mich dafür auf Arbeiten des österreichischen Zoologen Karl von Frisch (1886-1982), dessen Studien zur „Tanzsprache der Bienen“ bis heute wegweisend sind. Im Mittelpunkt steht eine Beobachtung von Frischs im Sommer 1944, die seine Vorstellungen über die Kommunikation bei Bienen erheblich verändern sollte. Wie ich zeigen möchte, gewinnt diese Beobachtung ihren Charakter als Einschnitt erst im Ablauf verschiedener Auf- und Abschreibeoperationen. Grundsätzlich scheint von Frisch Schreiben als ein Verfahren gebraucht zu haben, mit dem die konkrete Bedeutung des Aufgezeichneten zunächst offen gelassen und erst im Rückgriff näher fixiert wird. Das Beispiel macht allgemeiner darauf aufmerksam, dass schriftliche Aufzeichnungen, vom Beobachtungsjournal bis zum Entwurf einer Publikation, als Instrument unter anderen Instrumenten an der Formierung wissenschaftlicher Erkenntnisse beteiligt sind.
Escribir es una de las actividades más corrientes del investigador. Me propongo estudiar el papel que pueden desempeñar las notas en el proceso de investigación, estudiando los trabajos del zoólogo austríaco Karl von Frisch (1886-1982), cuya definición de la "danza de las abejas" no ha perdido nada de actualidad. Los conocimientos adquiridos durante el verano de 1944, condujeron a von Frisch a modificar sensiblemente su concepción del lenguaje de las abejas. Esta ruptura conceptual se produjo, no obstante, en (y a través de) la transformación de las notas copiadas y reescritas a partir de la notas tomadas durante la observación de las abejas. Parece pues que von Frisch se sirvió de la escritura para determinar a posteriori la significación concreta que había permanecido indeterminada en las notas tomadas precedentemente. Este ejemplo ilustra de una manera más general el hecho de que la escritura (desde el registro de observaciones hasta los esbozos últimos de una publicación) constituye uno de los instrumentos asociados a la génesis del conocimiento científico.

Scrivere è una delle più naturali attività degli studiosi. Si propone, qui, la considerazione del ruolo che nel processo di ricerca possono acquisire le note, studiando il lavoro dello zoologo austriaco Karl von Frisch (1886-1982), la cui definizione della "danse des abeilles" rimane attuale. L'attenzione si concentra su un'osservazione di von Frisch, dell'estate del 1944, che lo avrebbe obbligato a mutare sensibilmente le sue idee intorno al linguaggio delle api. Questo strappo concettuale si è, tuttavia, verificato nell'atto di copiare e riscrivere, modificandole, delle note prese durante l'osservazione delle api. Von Frisch sembra così essersi servito della scrittura per determinare in un secondo tempo il significato concreto, rimasto peraltro indefinito nelle note precedentemente prodotte. Questo esempio chiarisce, in senso più generale, come la scrittura (dalla fase di registrazione delle osservazioni al progetto definitivo di una pubblicazione) sia uno degli strumenti connessi alla genesi della conoscenza scientifica.

A escrita é uma das actividades mais comuns dos cientistas. Proponho-me considerar o papel dos apontamentos no processo de investigação, estudando os trabalhos do zoólogo austríaco Karl von Frisch (1886-1982), cuja definição da "dança das abelhas" não perdeu a actualidade. Foram dados obtidos no verão de 1944 o permitiram a von Frisch alterar significativamente a sua concepção da linguagem das abelhas. Esta mudança de ideias ocorreu durante (e por causa de) a transformação de apontamentos copiados e reescritos a partir de notas tomadas durante a observação das abelhas. Von Frisch parece ter-se servido dessa reescrita para determinar, a posteriori, o significado concreto que ficara impreciso na tomada inicial dos apontamentos. Este exemplo mostra de modo mais geral que a escrita (desde o registo de observações ao projecto final de publicação) é um instrumento pertinente na génese do conhecimento científico. 